\title{
A Variational Formulation and Analysis of Polar Orthotropic Circular Plates Using an Energy Principle, Part I: Thin Plates
}

\author{
Halil Ozer \\ Zonguldak Karaelmas University, Mechanical Engineering Department, 67100 Zonguldak, Turkey, \\ Email: halil_ozer@hotmail.com
}

\begin{abstract}
This paper concerns the bending analysis of an axisymmetric polar orthotropic thin circular plate (solid or annular) subjected to thermal or mechanical loading. Direct variational methods such as the Rayleigh-Ritz, the Galerkin, the Kantorovich and the Treftz have usually been employed to solve plate problems. In this study, the variational (energy) principle is used to derive the governing differential equations and the boundary conditions of the plate by using the rules of the calculus of variations. The set of differential equations derived from the variational process are solved simultaneously. The accuracy of the present formulation is demonstrated by the problems for which exact solutions are available, and the problems whose isotropic solutions are available while polar orthotropic solutions are not available. It is shown that the principle is useful to get solutions easily compared with the direct variational methods.
\end{abstract}

Keywords: Circular plates, polar orthotropy, calculus of variation

\section{INTRODUCTION}

The circular plate of anisotropic or isotropic nature is an important research subject due to its relation to ocean, aerospace, mechanical and civil engineering applications. It is used in pump diaphragms and clutches, and is also an essential part of numerous engineering systems. Circular concrete slabs are reinforced with steel in the radial and circumferential directions. Circular plates composed of cuts of wood have naturally material orthotropy.

Various numerical methods are adopted for solving isotropic or orthotropic circular plate problems. Meleshko /1/ gives a historical overview of some topics related to the classical 2D biharmonic problems in some studies concerning bending of thin elastic circular plates. Salem et al. /2/ presented approximate series solutions for orthotropic circular plate. Huann et al. $/ 3$ / obtained integral representations of solutions for circular plate problems. Melnikov /4/applied the Green's function to thin circular plates. Elettary /5/ used the eigenfunction expansion to obtain the deflection of a circular plate under non-uniform load. Recently, we studied an axisymmetrical bending problem of nonlinearly tapered annular plate with free edge by using a relationship between Mindlin and Kirchhoff bending solutions $/ 6 /$.

The term "variational formulation" applies to a wide spectrum of concepts having to do with weak, generalized, or direct variational formulation of boundary and initial value problems /7/. The direct variational methods such as the Rayleigh-Ritz, the Galerkin, the Kantorovich and the Treftz have usually been employed to solve the plate problems. 
Bending solutions of thin circular or rectangular plates by the use of the direct variational methods can be found in many standard texts. Liew and Yang /8/ studied free vibration of circular plates by using the Ritz method. Kang et al /9/ analyzed free vibration of the polar orthotropic circular plate using the Ritz Method. Pavlovic et al. /10/ applied a variational symbolic solution to bending of clamped orthotropic rectangular plates using the Galerkin Method. However, the direct variational methods require the introduction of terms with unknown coefficients in order to improve the accuracy that makes the mathematical analysis more complicated. Although there is an extensive literature on circular plates, a rather limited amount of literature is available on application of the variational principle to the polar orthotropic circular plates. In this context, Pedregal and Donoso /11/ analyzed the one dimensional situation through a variational formulation for a design problem of a plate with variable thickness. Najafizadeh and Eslami /12/ studied the thermoelastic buckling of a circular orthotropic composite plate. They derived the equilibrium and stability equations via variational formulations.

In this study, we use the principle of minimum potential energy in conjunction with the variational calculus. When energy principles are applied to continuum problems, the appropriate mathematical tool is the calculus of variations. The variational calculus is concerned with the extreme conditions of certain definite integral called functional. The superiority of the variational calculus is that it provides both the governing differential equations together with the boundary conditions of the problem. Many more calculations would be necessary for the use of such direct variational methods without the use of the variational principle. The variational formulation obtained for the problem was according to the rules of the calculus of variations. The application of the principle to the derivation of the equilibrium equation and boundary conditions is presented. The principle is applied to the sample problems concerning the thermally or mechanically loaded polar orthotropic circular plates in order to verify its appropriateness. A comparison is made between the results obtained in this study and the results reported in literature.

\section{BASIC EQUATIONS}

For an annular (or circular) plate, shown in Fig. 1, elastic strains may be obtained through Hooke's law. The strain displacement equations take the following form for the axisymmetric case /13/

$$
\begin{aligned}
& \varepsilon_{r}=\frac{d u}{d r}=-z w^{\prime \prime}(r) \\
& \varepsilon_{\theta}=\frac{u}{r}=-z \frac{1}{r} w^{\prime}(r)
\end{aligned}
$$

where $w$ is the transverse deflection of the plate. In Eqs. (1)-(2), $w^{\prime}(r)$ and $w^{\prime \prime}(r)$ are equal to $d w / d r$ and $d^{2} w / d r^{2}$. respectively. As shown in Fig. 1, the principal material axes are assumed to coincide with the polar coordinates $(r, \theta)$. 

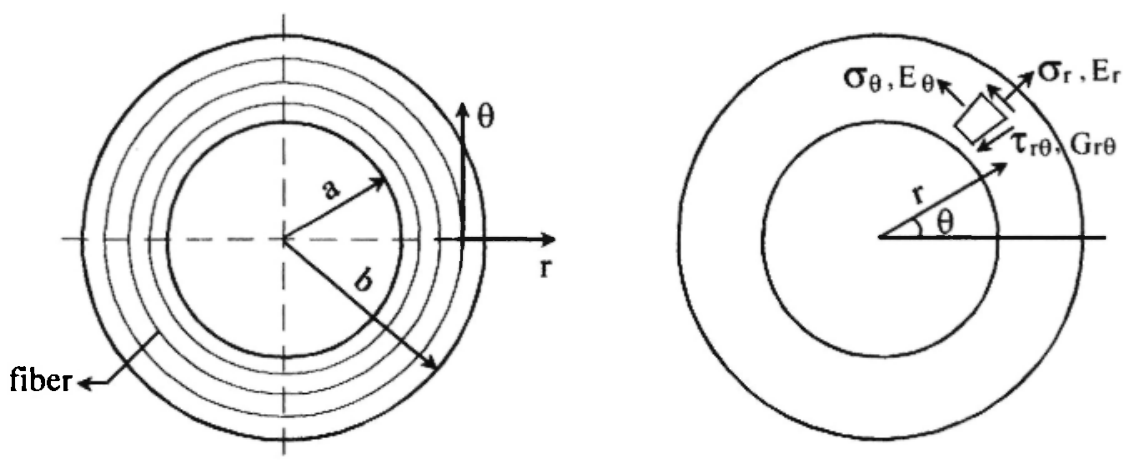

Fig. 1: Polar orthotropic annular plate

The plane-stress thermoelastic constitutive equations can be written for an orthotropic material as follows:

$$
\left\{\begin{array}{c}
\sigma_{r} \\
\sigma_{\theta} \\
\tau_{r \theta}
\end{array}\right\}-\left[\begin{array}{ccc}
Q_{11} & Q_{12} & 0 \\
Q_{12} & Q_{22} & 0 \\
0 & 0 & Q_{66}
\end{array}\right]\left\{\begin{array}{c}
\varepsilon_{r}-\alpha_{r} \Delta T \\
\varepsilon_{\theta}-\alpha_{\theta} \Delta T \\
\gamma_{r \theta}
\end{array}\right\}
$$

where $\Delta T(r, z)$ is the temperature increment, $\alpha_{r}$ and $\alpha_{\theta}$ are the coefficients of thermal expansion. $Q_{i j}$ represent the elastic reduced stiffness coefficients, and are defined as follows:

$$
\begin{aligned}
& Q_{11}=\frac{E_{r}}{1-v_{r \theta} v_{\theta r}}, Q_{12}=\frac{v_{\theta r} E_{r}}{1-v_{r \theta} v_{\theta r}} \\
& Q_{22}=\frac{E_{\theta}}{1-v_{r \theta} v_{\theta r}}, Q_{66}=G_{r \theta}
\end{aligned}
$$

where $v_{r \theta}$ and $v_{\theta r}$ are the Poisson's ratios. $E_{r}$ and $E_{\theta}$ are the Young's moduli with respect to the radial and circumferential directions, respectively. $G_{r \theta}$ is the shear modulus. The strain energy for circular plate may be written as follows:

$$
U=\iiint \varepsilon_{i j} \sigma_{i j} r d r d \theta d z
$$

The total potential energy is defined as the difference between the strain energy and the potential energy of the applied loads as

$$
\pi \equiv U+W
$$

where $W$ is the potential energy of the applied loads. The total potential energy $\pi$ may be written for an annular plate on an elastic foundation of modulus $\lambda$, which is subjected to uniformly distributed transverse load $q$, bending moment $M_{r}$, and shear force $Q_{r}$, by substituting Eqs. (1), (2) and (3) into (6), and by using Eq. (5) as follows 


$$
\begin{aligned}
\pi= & \int_{R}\left[\frac{D_{r}}{2}-w^{\prime \prime}(r)^{2}+D_{r \theta} \frac{1}{r} w^{\prime}(r) w^{\prime \prime}(r)+\frac{D_{\theta}}{2} \frac{1}{r^{2}} w^{\prime}(r)^{2}+M_{r}^{T} w^{\prime \prime}(r)+\frac{1}{r} M_{\theta}^{T} w^{\prime}(r)\right. \\
& \left.+\frac{1}{2} \lambda w(r)^{2}\right] r d r d \theta-\int_{R} q(r) w(r) r d r d \theta-\int_{S_{1}} Q_{r} w(r) d s+\int_{S_{2}} M_{r} w^{\prime}(r) d s
\end{aligned}
$$

where $R$ denotes the midplane of the plate. $S_{1}$ and $S_{2}$ denote the boundary parts on which the shear forces and the bending moments are specified. $d s$ is equal to $r d \theta . D_{i j}$ are the bending stiffnesses of the plate and are defined in terms of $Q_{i j}$ as

$$
D_{i j}=\frac{h^{3}}{12} Q_{i j}
$$

where $h$ is the plate thickness. $M_{r}^{T}$ and $M_{\theta}^{T}$ are the thermal moments with respect to $r$ and $\theta$, respectively and can be written as

$$
\begin{aligned}
& M_{r}^{T}=\left(Q_{11} \alpha_{r}+Q_{12} \alpha_{\theta}\right) \int_{-h / 2}^{+h / 2} \Delta T(r, z) z d z \\
& M_{\theta}^{T}=\left(Q_{12} \alpha_{r}+Q_{22} \alpha_{\theta}\right) \int_{-h / 2}^{+h / 2} \Delta T(r, z) z d z
\end{aligned}
$$

Bending moments and shear force may be written for the polar orthotropic case as

$$
\begin{aligned}
& M_{r}=-\left[D_{r} w^{\prime \prime}(r)+D_{r \theta} \frac{1}{r} w^{\prime}(r)\right]-M_{r}^{\prime} \\
& M_{\theta}=-\left[D_{r} w^{\prime \prime}(r)+D_{\theta} \frac{1}{r} w^{\prime}(r)\right]-M_{\dot{U}}^{\prime} \\
& Q_{r}=-D_{r} w^{\prime \prime \prime}(r)-\frac{1}{r} D_{r} w^{\prime \prime}(r)+\frac{1}{r^{2}} D_{\theta} w^{\prime}(r)-\frac{1}{r}\left[\frac{d}{d r}\left(r M_{r}^{T}\right)-M_{\theta}^{T}\right]
\end{aligned}
$$

\section{VARIATIONAL FORMULATION OF THE PROBLEM}

We apply the formal procedure of the calculus of variations to the total potential energy functional to obtain the governing differential equations and the appropriate boundary conditions. Firstly, we can write the total potential energy of the circular plate as follows $/ 7,13,14,15 /$ :

$$
\pi=\int_{R} F\left(r, w, w^{\prime}, w^{\prime \prime}\right) r d r d \theta
$$


where the total potential energy functional, $F$, is a function of the dependent variable $w(r)$ and its derivatives. We consider perturbations of the transverse deflection curve as follows:

$$
\ddot{w}(r)=w(r)+\delta w(r)=w(r)+\varepsilon \eta(r)
$$

where the operator $\delta$ is called the variational operator. $\varepsilon \eta(r)$ is a variation of $w(r)$. The variation $\delta w$, called the first variation of $w(r)$, must be consistent with the geometric constraint of the problem. $\eta(r)$ is an arbitrary function satisfying end point conditions of the problem. $\varepsilon$ is a small arbitrary number. $\pi(\varepsilon)$ attains a minimum at $\varepsilon=0$, at which $\hat{w}(r)=w(r)$.

The principle of the potential energy is one of the most important variational (or energy) principle in theory of elasticity, which can be used to find equilibrium conditions through minimizing the total potential energy of the system. The principle of minimum potential energy can be defined with the first variation of the total potential energy as

$$
\delta \pi \equiv \delta(U+W)=0
$$

where $\delta \pi$ represents the first variation of $\pi$. When $\delta \pi-0$, this condition make the potantial energy a stationary value. For stable equilibrium, this stationary value is a minimum. The equation, $\delta \pi-0$, means that if we add any small change $\delta w$ to the actual $w$, the change in the total potential energy will be zero. The first variation of $\pi$ can be written as the variational integral of the energy functional as

$$
\delta \pi=\int_{R} \delta F r d r d \theta=0
$$

The energy functional $F$ is obtained from Eq. (7). $\delta F$ is obtained by using the Taylor expansion in terms of Eq. (12) as follows:

$$
\int_{R} \delta F r d r d \theta=2 \pi \int_{a}^{b}\left[\varepsilon \eta \frac{\partial}{\partial w}+\varepsilon \eta^{\prime} \frac{\partial}{\partial w^{\prime}}+\varepsilon \eta^{\prime \prime} \frac{\partial}{\partial w^{\prime \prime}}\right] F\left(r, w, w^{\prime}, w^{\prime \prime}\right) r d r=0
$$

where $a$ is the inner radius and $b$ the outer radius of the annular plate. Here $\varepsilon \eta(r)=\delta w(r), \varepsilon \eta^{\prime}(r)=\delta w^{\prime}(r)$, and $\varepsilon \eta^{\prime \prime}(r)=\delta w^{\prime \prime}(r)$. Then, Eq. (15a) may be expressed as

$$
\delta \pi=2 \pi \int_{a}^{b}\left[\frac{\partial F}{\partial w} \delta w+\frac{\partial F}{\partial w^{\prime}} \delta w^{\prime}+\frac{\partial F}{\partial w^{\prime \prime}} \delta w^{\prime \prime}\right] r d r=0
$$

where Eq. (15) includes derivatives of the functional and yields the Euler equations together with the boundary conditions of the problem. We carry out the successive integration by parts or use the gradient theorem on second and third terms in Eq. (15), then collect the coefficients of $\delta w$ and $\delta w^{\prime}$ separately and set its first variation equal to zero, finally the condition $\delta \pi=0$ leads to 


$$
\begin{aligned}
\delta \pi & =2 \pi \int_{r}^{b}\left\{D_{r} w^{I V}(r)+\frac{2}{r} D_{r} w^{\prime \prime}(r)-\frac{1}{r^{2}} D_{\theta} w^{\prime \prime}(r)+\frac{1}{r^{3}} D_{\theta} w^{\prime}(r)+\lambda w(r)-q+\frac{1}{r} \frac{d}{d r}\left[\frac{a}{d r}\left(r M_{r}^{T}\right)-M_{\theta}^{T}\right]\right\} \delta w(r) d r \\
& +\left.2 \pi\left\{-D_{r} r w^{\prime \prime}(r)-D_{r} w^{\prime \prime}(r)+D_{\theta} \frac{1}{r} w^{\prime}(r)+\left[M_{\theta}^{T}(r)-M_{r}^{T}(r)\right]-r \frac{d M_{r}^{T}}{d r}-r Q_{r}\right\} \delta w(r)\right|_{a} ^{b} \\
& +\left.2 \pi\left\{D_{r} r w^{\prime \prime}(r)+D_{r} w^{\prime}(r)+r M_{r}^{T}(r)+r M_{r}\right\} \delta w^{\prime}(r)\right|_{a} ^{b}=0
\end{aligned}
$$

Eq. (16) leads to the following relations

$$
\begin{aligned}
& 2 \pi\left\{D_{r} w^{\prime V}(r)+\frac{2}{r} D_{r} w^{\prime \prime \prime}(r)-\frac{1}{r^{2}} D_{\theta} w^{\prime \prime}(r)+\frac{1}{r^{2}} D_{\theta} w^{\prime}(r)+\lambda w(r)-q+\frac{1}{r} \frac{d}{d r}\left[\frac{d}{d r}\left(r M_{r}^{T}\right)-M_{\theta}^{T}\right]\right\} \delta w(r)=0 \\
& 2 \pi\left\{-D_{r} b w^{\prime \prime}(b)-D_{r} w^{\prime \prime}(b)+D_{\theta} \frac{1}{b} w^{\prime}(b)+\left[M_{\theta}^{T}(b)-M_{r}^{T}(b)\right]-\left.b \frac{d M_{r}^{T}}{d r}\right|_{r=b}-b Q_{b}\right\} \delta w(b)=0 \\
& 2 \pi\left\{D_{r} a w^{\prime \prime}(a)+D_{r} w^{\prime \prime}(a)-D_{\theta} \frac{1}{a} w^{\prime}(a)-\left[M_{\theta}^{T}(a)-M_{r}^{T}(a)\right]+\left.a \frac{d M_{r}^{T}}{d r}\right|_{r=a}-a Q_{a}\right\} \delta w(a)=0 \\
& 2 \pi\left\{D_{r} b w^{\prime \prime}(b)+D_{r \theta} w^{\prime}(b)+b M_{r}^{T}(b)+b M_{b}\right\} \delta w^{\prime}(b)=0 \\
& 2 \pi\left\{-D_{r} a w^{\prime \prime}(a)-D_{r} w^{\prime}(a)-a M_{r}^{T}(a)-a M_{a}\right\} \delta w^{\prime}(a)=0
\end{aligned}
$$

where $\delta w(a), \delta w(b), \delta w^{\prime}(a)$ and $\delta w^{\prime}(b)$ give the essential boundary conditions of the plate. The expressions inside the braces of Eqs. (17b) to (17e) give natural boundary condition of the plate. Since the Eq. (17a) must be zero for an arbitrary deflection $\delta w$, the expression inside the braces in Eq. (17a) gives the governing differential equation of the problem as

$D_{r} w^{N}(r)+\frac{2}{r} D_{r} w^{\prime \prime \prime}(r)-\frac{1}{r^{2}} D_{\theta} w^{\prime \prime}(r)+\frac{1}{r^{3}} D_{\theta} w^{\prime}(r)+i w(r)=q-\frac{1}{r} \frac{d}{d r}\left[\frac{d}{d r}\left(r M_{r}^{T}\right)-M_{\theta}^{T}\right]$

It should be noted that the expressions inside the braces of Eqs. (17b) and (17c) give the shear forces at $r=a$ and $r=b$, respectively. It can be easily seen that the expressions inside the braces of Eqs. (17b) and (17c) are identical to Eq. (10c). The expressions inside the braces of Eqs. (17d) and (17e) also give the radial bending moments at $r=a$ and $r=b$, respectively. The expressions inside the braces of (17d) and (17e) are also identical to Eq. (10a).

Consequently, we demonstrated that the variational approach yields the boundary value problem. To solve the boundary value problem, the governing differential equation must be evaluated by using essential and natural boundary conditions.

\section{ILLUSTRATIVE EXAMPLES: THERMALLY OR MECHANICALLY LOADED SOLID CIRCULAR PLATES}

In order to verify the usefulness of the principle, this method is applied to four sample problems concerning the 
polar orthotropic solid circular plates. Circular plates are considered to be subjected to thermal or mechanical loading. The temperature effect is examined at the first two examples and the mechanical effect is examined at the last two examples. The condition, $\delta \pi=0$, is used to get the critical curve of the transverse deflection of the plate.

Case 1: Thermally loaded solid circular plate with simply supported edge:

An aircraft window can be modelled as a simply supported solid circular plate (Fig. 2). It is subjected to a uniform temperature $T_{1}$ at the lower surface and $T_{2}$ at the upper surface. It is also assumed that the temperature is varying linearly with thickness and that $T_{1}>T_{2}$. The temperature increment is given as follows /16/:

$$
\Delta T=\frac{1}{2}\left(T_{1}+T_{2}\right)+\frac{1}{2}\left(T_{1}-T_{2}\right) \frac{z}{h / 2}
$$

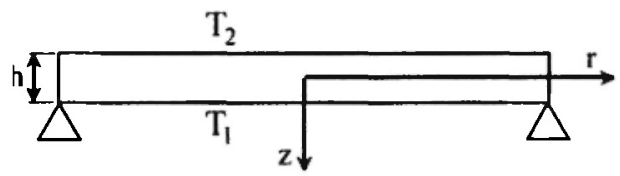

Fig. 2: Model of an aircraft window

The boundary conditions are defined as follows: $a Q_{a}=0, w^{\prime}(a)=0\left[\delta w^{\prime}(a)=0\right]$ at $a=0$, and $w(b)=0$ $[\delta w(b)=0], b M_{b}=0$ at $r=b$. We obtain the set of equations by using Eqs. (17) as follows

$$
\begin{aligned}
& D_{r} w^{\prime N}(r)+\frac{2}{r} D_{r} w^{\prime \prime \prime}(r)-\frac{1}{r^{2}} D_{\theta} w^{\prime \prime}(r)+\frac{1}{r^{3}} D_{\theta} w^{\prime}(r)+\frac{1}{r} \frac{d}{d r}\left[\frac{d}{d r}\left(r M_{r}^{T}\right)-M_{\theta}^{T}\right]=0 \\
& w(b)=0 \\
& a D_{r} w^{\prime \prime \prime}(a)+D_{r} w^{\prime \prime}(a)-\frac{1}{a} D_{\theta} w^{\prime}(a)=0 \\
& D_{r} b w^{\prime \prime}(b)+D_{r} w^{\prime}(b)+b M_{r}^{T}(b)=0 \\
& w^{\prime}(a)=0
\end{aligned}
$$

It can be seen that Eq. (20c) is equivalent to the condition that the shear force vanishes at $r=0$. Eq. (20d) means that the radial bending moment is zero at $r=b$. Finally, this problem is reduced to the simultaneous solution of the set of differential equations. It is generally very difficult to solve the equations derived from the variational process. This is the domain of the ordinary calculus. We used the computer algebra code Mathematica to solve simultaneously the set of differential equations. By solving the Eq. (20a) simultaneously with the set of Eqs. (20b)-(20c), which are the essential and natural boundary conditions of the problem, and then by applying the limit operation to the obtained expression as $a \rightarrow 0$, the deflection function is finally reduced to

$$
w(r)=\frac{\left(\alpha_{r}+v_{\theta r} \alpha_{\theta}\right)}{\left(v_{\theta r}+\sqrt{k}\right)} \frac{b^{1-k}\left(b^{i+1}-r^{k+1}\right)}{h(1+k)}\left(T_{1}-T_{2}\right)
$$


where

$$
\begin{aligned}
& k=\sqrt{\frac{D_{\theta}}{D_{r}}}=\sqrt{\frac{E_{\theta}}{E_{r}}} \\
& \frac{v_{r \theta}}{E_{r}}=\frac{v_{\theta r}}{E_{\theta}}
\end{aligned}
$$

To our knowledge, Eq. (21) is not available in the open literature and is derived in this study. Therefore, the deflection function of the isotropic plate can play a key role to check the accuracy of the result of polar orthotropic case given by Eq. (21). For this purpose, we use the transformations between the polar orthotropic and isotropic plate as follows:

$$
\begin{aligned}
& v_{r \theta}=v_{\theta r}=v \\
& \alpha_{r}=\alpha_{\theta}=\alpha \\
& D_{r}=D_{\theta}=D, D_{12}=v D, D=E h^{3} / 12\left(1-v^{2}\right) \\
& M_{r}^{T}=M_{\theta}^{T}=M_{T} /(1-v), M_{T}=E \alpha \int \Delta T(r, z) z d z
\end{aligned}
$$

where $E, v, D, \alpha$ and $M_{T}$ define the Young's modulus, Poisson's ratio, bending stiffness, the coefficient of thermal expansion, and the thermal stress resultant of the isotropic plate, respectively. Consequently, we obtained the deflection function for the isotropic case rearranging the Eqs. (20) through the transformations given in Eq. (23) or by setting $k=1$, which corresponds to the isotropic case, in Eq. (21) as follows:

$$
w(r)=\frac{\alpha\left(b^{2}-r^{2}\right)}{2 h}\left(T_{1}-T_{2}\right)
$$

The expression given in Eq. (24) is the same with that of the reported in $/ 16 \%$

Case 2: Thermally loaded solid circular plate with clamped edge:

Secondly, we use the same temperature function given in Eq. (19). The boundary conditions are defined as follows: $a Q_{a}=0, w^{\prime}(a)=0 / \delta w^{\prime}(a)=0 /$ at $a=0$, and $w(b)=0, w^{\prime}(b)=0 / \delta w^{\prime}(b)=0 /$ at $r=b$. Then, we obtain the set of equations by using Eqs. (17) as follows

$$
\begin{aligned}
& D_{r} w^{\prime V}(r)+\frac{2}{r} D_{r} w^{\prime \prime \prime}(r)-\frac{1}{r^{2}} D_{\theta} w^{\prime \prime}(r)+\frac{1}{r^{3}} \bar{D}_{\theta} w^{\prime}(r)+\frac{1}{r} \frac{d}{d r}\left[\frac{d}{d r}\left(r M_{r}^{T}\right)-M_{\theta}^{T}\right]=0 \\
& w(b)=0 \\
& a D_{r} w^{\prime \prime \prime}(a)+D_{r} w^{\prime \prime}(a)-\frac{1}{a} D_{\theta} w^{\prime}(a)-\left[M_{\theta}^{T}(a)-M_{r}^{T}(a)\right]=0 \\
& w^{\prime}(b)=0 \\
& w^{\prime}(a)=0
\end{aligned}
$$


It is seen that Eq. (20c) is equivalent to the condition that the shear force is equal to zero at $r=0$. By solving Eq. (25a) simultaneously with the set of Eqs. (25b) to (25e), and then by applying the limit operation to the expression as $a \rightarrow 0$, we have

$$
w(r)=0
$$

The same problem is solved just for isotropic solid circular plate in $/ 17 /$. The deflection function is found to be zero. We also found the same function for polar orthotropic solid circular plate.

Case 3: Uniformly transverse loaded solid circular plate with clamped edge:

Thirdly, we studied the deflection function of solid circular plate with clamped edge under a uniformly distributed load $q$. The boundary conditions are defined as follows: $a Q_{a}=0, w^{\prime}(a)=0 / \delta w^{\prime}(a)=0 /$ at $a=0$, and $w(b)=0$, $w(b)=0 / \delta w(b)=0 /$ at $r=b$. We obtain the set of equations by using Eqs. (17) as follows:

$$
\begin{aligned}
& D_{r} w^{I V}(r)+\frac{2}{r} D_{r} w^{\prime \prime}(r)-\frac{1}{r^{2}} D_{\theta} w^{\prime \prime}(r)+\frac{1}{r^{3}} \nu_{0} w^{\prime}(r)-q=0 \\
& w(b)=0 \\
& D_{r} a w^{\prime \prime \prime}(a)+D_{r} w^{\prime \prime}(a)-D_{\theta} \frac{1}{a} w^{\prime}(a)=0 \\
& w^{\prime}(b)=0 \\
& w^{\prime}(a)=0
\end{aligned}
$$

It is seen that Eq. (27c) corresponds to the condition that the shear force is equal to zero at $r=0$. By solving Eq. (27a) simultaneously with the set of Eqs. (27b)-(27e), and then applying the limit operation to the expression as $a \rightarrow 0$, the deflection function is simplified to the following equation:

$$
u(r)=\frac{q b^{4}}{8\left(9-k^{2}\right)(1+k) D_{r}}\left[3-k-4\left(\frac{r}{b}\right)^{1+k}+(1+k)\left(\frac{r}{b}\right)^{4}\right]
$$

We obtain the deflection function for isotropic case through rearranging Eq. (28) by setting $k=1$, as follows

$$
w(r)=\frac{q b^{4}}{64 D}\left[1-\left(\frac{r}{b}\right)^{2}\right]^{2}
$$

The expressions obtained in Eq. (28) and Eq. (29) are the same expressions reported in /18/ and /19/, respectively. It is seen that the deflection expression obtained in Eq. (28) is going to the limit and can not be applied to materials having $k=3$. The appropriate expression may be obtained through the limiting value of the function. We can use the limit operator for detlection function as follows 


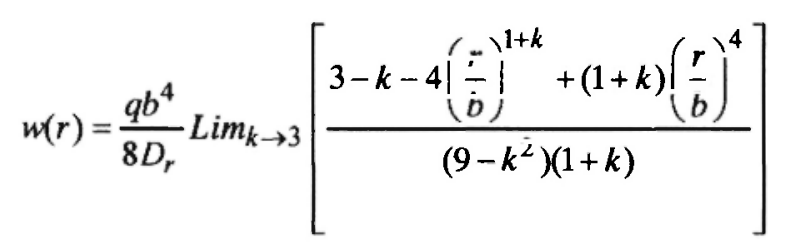

Then, we obtained the function for $k=3$ as

$$
w(r)=\frac{q b^{4}}{192 D_{r}}\left[1-\left(\frac{r}{b}\right)^{4}+4\left(\frac{r}{b}\right)^{4} \ln \frac{r}{b}\right]
$$

Therefore, we overcome the singularity at $k=3$. Consequently, the formula are applicable to all values of $k$ by selecting the appropriate function.

Case 4: Central Point loaded solid circular plate with clamped edge:

Finally, we studied the deflection function of solid circular plate with clamped edge under concentrated point load $P$ at the center of the plate. The boundary conditions are defined as follows: $a Q_{a}=P / 2 \pi, w(a)=0 / \delta w(a)=0 /$ at $a=0$ and $w(b)=0, w(b)=0 / \delta w(b)=0 /$ at $r=b$. We obtain the set of equations by using Eqs. (17) as follows

$$
\begin{aligned}
& D_{r} w^{I V}(r)+\frac{2}{r} D_{r} w^{\prime \prime \prime}(r)-\frac{1}{r^{2}} D_{\theta} w^{\prime \prime}(r)+\frac{1}{r^{3}} D_{\theta} w^{\prime}(r)=0 \\
& w(b)=0 \\
& D_{r} a w^{\prime \prime \prime}(a)+D_{r} w^{\prime \prime}(a)-\because=\hat{o} \frac{1}{a} w^{\prime}(a)=\frac{P}{2 \pi} \\
& w^{\prime}(b)=0 \\
& w^{\prime}(a)=0
\end{aligned}
$$

where Eq. (32c) means that the shear force is equivalent to $P / 2 \pi a$ at the center of the plate. By solving Eq. (32a) simultaneously with the set of Eqs. (32b)-(32e), and then by applying the limit operation to the expression as $a \rightarrow 0$, the deflection function is obtained as follows

$$
w(r)=\frac{P b^{2}}{4 \pi\left(1-k^{2}\right)(k+1) D_{r}}\left[1-k+(k+1)\left(\frac{r}{b}\right)^{2}-2\left(\frac{r}{b}\right)^{k+1}\right]
$$

It is seen that the deflection expression obtained in Eq. (33) is going to the limit at $k=1$. To overcome the singularity at $k=1$, we can use the limit operator for deflection expression as follows

$$
\omega(r)=\frac{P b^{2}}{4 \pi D_{r}} \operatorname{Lim}_{k \rightarrow 1}\left[\frac{1-k+(k+1)\left(\frac{r}{b}\right)^{2}-2\left(\frac{r}{b}\right)^{k+1}}{\left(1-k^{2}\right)(k+1)}\right]
$$

Therefore, we obtained the expression for isotropic plate as follows 


$$
w(r)=\frac{P b^{2}}{16 \pi D}\left[1-\left(\frac{r}{a}\right)^{2}+2\left(\frac{r}{a}\right)^{2} \operatorname{In} \frac{r}{a}\right]
$$

The expressions given in Eq. (33) and Eq. (35) are exactly the same expressions reported in /18/.

\section{CONCLUSION}

In this study, the variational formulation is obtained for polar orthotropic thin annular and solid circular plates by using the rules of the calculus of variations. The application of the variational principle to the formulation of the problem yields the governing differential equations together with the boundary conditions. Thus, the boundary value problem is obtained directly and easily from the variational principle. Illustrative examples are given for solid circular plates to check the accuracy of the deflection functions. It is shown that the deflection functions are exactly the same as reported in literature. These results also indicate the validity of the proposed variational formulations for polar orthotropic circular plates and confirm the fact that the expression of the total potential energy is correct. Otherwise, the first variation of the total potential energy would not lead to the correct equilibrium equation, essential and natural boundary conditions and, the deflection function of the plate with incorrect relations. The considered problems in this study are the mixed boundary value problems in which both essential and natural boundary conditions are used. Although this study concerned with the deflection analysis of the plate, stress resultants and stresses can be obtained easily by using Eq. (10) and Eq. (3), respectively. Consequently, the results of the present paper indicate that variational principle is the powerful tool to formulate and get solutions easily for many problems of circular plates in terms of variational statements. Therefore, it is convenient to rely on energy considerations in order to discuss the displacement, stress resultant and stress relations in plate problems. It can also be noted that the deflection expressions are independent of Elastic modulus and Poisson's ratio for isotropic circular plates, while it is related to moduli of elasticity $\left(E_{r}, E_{\theta}\right)$ and Poisson's ratio $\left(v_{r \theta}\right.$ or $\left.v_{\theta r}\right)$ for the polar orthotropic circular plates.

This study provides some indication of how the energy principle may be applied to the problems of polar orthotropic thin circular plates by solving simultaneously the set of differential equations derived from the variational process. The similar procedure can be employed for the case of thick circular (or an annular) plate by using any shear deformation theory and for the case of plate with statically indeterminate boundary conditions.

\section{REFERENCES}

1. V.V. Meleshko. Selected topics in the history of the two-dimensional biharmonic problem, Appl. Mech. Rev., 56, 33-85 (2003).

2. J.A. Salem and J.M. Manderscheid. Stresses in a thin aelotropic plate subjected to uniform lateral load, J. Phys. DAppl. Phys., 36, 2730-2737 (2003).

3. P.G. Hunan and P.R. China. The integral representation of solutions for circular plate problems without any hold, Acta Mechanica, 163, 173-182 (2003). 
4. Y.A. Melnikov. Green's function of a thin circular plate with elastically supported edge, Eng. Anal. Bound. Elem., 25, 669-676 (2001).

5. M.A. Elattary. Application of eigenfunction expansion to elastic circular plates, J. Phys. D-Appl. Phys., 22, $302-$ 304 (1989).

6. H. Ozer. A Comparative analysis of Mindlin and Kirchhoff bending solutions for nonlinearly tapered annular plate with free edges, Arch. Appl. Mech., 22, 393-405 (2007).

7. J.N. Reddy. Energy and Variational Methods in Applied Mechanics, John Wiley \& Sons, 1984.

8. K.M. Liew and B. Yang. Three dimensional elasticity solutions for free vibrations of circular plates: a polynomials - Ritz analysis, Comp. Meth. Appl. Mech. Eng., 175, 189-201 (1999).

9. W. Kang, N.H. Lee, S. Pang and W.Y. Chung. Approximate closed form solutions for free vibration of polar orthotropic circular plates, Appl. Acoust., 66, 1162-1179 (2005).

10. F.C. Mbakogu and M.N. Pavlović. Bending of clamped orthotropic rectangular plates: a variational symbolic solution, Comput. Struct. 77, 117-128 (2000).

11. P. Pedregal and A. Donoso. Optimal design of a plate of variable thickness: a variational approach in dimension one, Comput. Appl. Math., 22, 75-89 (2003).

12. M.M. Najafizadeh and M.R. Eslami. Thermoelastic stability of orthotropic circular plates, J. Therm. Stresses, 25, 985-1005 (2002).

13. J.N. Reddy. Theory and Analysis of Elastic Plates, Taylor \& Francis, 1999.

14. T.H. Richards,. Energy Methods in Stress Analysis, John Wiley \& Sons, 1977.

15. J.N. Reddy. Applied Functional Analysis and Variational Methods in Engineering, McGraw Hill, 1986.

16. A.C. Ugural. Stresses in Plates and Shells, McGraw Hill, 1999.

17. N. Noda, R.B. Hetnarski and Y. Tanigawa. Thermal Stresses, Lastran, 2000.

18. S.G. Lekhnitskii, S.W. Tsai and T. Cheron. Anisotropic Plates, Gordon and Breach Science Publishers, New York, 1968.

19. S.P. Timoshenko and S.W. Krieger. Theory of Plates and Shells, McGraw-Hill, 1959. 